\title{
SPIRAL ARM MORPHOLOGY OF NEARBY GALAXIES
}

\author{
Hong Bae AnN ${ }^{1}$ And Hyun-Rok LeE ${ }^{2}$ \\ ${ }^{1}$ Department of Earth Science Education, Pusan National University, Pusan 609-735, Korea \\ E-mail : hbann@pusan.ac.kr \\ ${ }^{2}$ Korea Institute of Science and Technology, Daejeon 136-791, Korea \\ E-mail : hyunrok@kaist.ac.kr \\ (Received May 1, 2013; Revised May 26, 2013; Accepted May 31, 2013)
}

\begin{abstract}
We analyze the spiral structure of 1725 nearby spiral galaxies with redshift less than 0.02 . We use the color images provided by the Sloan Digital Sky Survey. We determine the arm classes (grand design, multiple-arm, flocculent) and the broad Hubble types (early, intermediate, late) as well as the bar types (SA, SAB, SB) by visual inspection. We find that flocculent galaxies are mostly of late Hubble type while multiple-arm galaxies are likely to be of early Hubble type. The fractional distribution of grand design galaxies is nearly constant along the Hubble type. The dependence of arm class on bar type is not as strong as that of the Hubble type. However, there is about a three times larger fraction of grand design spirals in SB galaxies than in SA galaxies, with nearly constant fractions of multiple-arm galaxies. However, if we consider the Hubble type and bar type together, grand design spirals are more frequent in early types than in late types for SA and SAB galaxies, while they are almost constant along the Hubble type for SB galaxies. There are clear correlations between spiral structures and the local background density: strongly barred, early-type, grand design spirals favor high-density regions, while non-barred, late-type, flocculent galaxies are likely to be found in low-density regions.
\end{abstract}

Key words : galaxies: structure — galaxies: formation — galaxies: morphology — galaxies: general

\section{INTRODUCTION}

Spiral arms are the most prominent features of spiral galaxies. Broadly speaking, there are two types of spiral structures: grand design arms, and flocculent arms. Grand design arms are characterized by long symmetric arms, while flocculent arms are by chaotic, fragmented arms. Some galaxies show grand design arms in the inner parts, and multiple arms in the outer parts. Hence, we can divide spiral galaxies into three classes: grand design, multiple-arm, and flocculent spirals. The origin of the differences in the spiral arm structure is believed to be the different mechanisms of spiral arm formation. Conventionally, grand design arms have been explained in the framework of density wave theory (Lin \& Shu 1964), in particular, the modal theory (Bertin et al. 1989a,b) described well how the spiral arm structure is maintained and evolved. Instead, flocculent arms are explained by the stochastic self-propagated star formation theory proposed by Gerola \& Seiden (1978) and Seiden \& Gerola (1982).

There have been several studies on the correlation between spiral arm morphology and internal and external properties of spiral galaxies. Elmegreen \& Elmegreen (1982) introduced an arm classification system based on the regularity of the spiral arm structure, in which arm classes (AC) 1-4 represent flocculent

Corresponding Author: H. B. Ann galaxies, AC 5-9 multiple-arm galaxies and AC 10-12 grand design galaxies, respectively. However, most previous correlation studies divided their sample galaxies into two broad classes, namely flocculent galaxies (AC1-4) and grand design galaxies (AC5-12) because of a larger sample size for better statistics. There seems to be no correlation between the arm class and the Hubble type except for the deficit of grand design galaxies in Sd and Sm types (Elmegreen \& Elmegreen 1982). However, Elmegreen \& Elmegreen (1982) found that there is a correlation between the spiral arm structure and galaxy environment from binary (Turner 1976) and group galaxies (Turner \& Gott 1976) together with field galaxies selected from the RC2 (de Vaucouleurs, de Vaucouleurs, \& Corwin 1976). Elmegreen \& Elmegreen (1982) showed that the fraction of grand design galaxies in the field sample depends critically on the presence of bar, whereas galaxies in binary and group samples are not much affected by the presence of a bar. The grand design fraction of non-barred galaxies in the field sample is about one third, while that of barred galaxies is about two thirds, regardless of their environment. However, the previous results have poor statistical significance because the number of galaxies in each bin is too small.

The arm classification system was slightly modified by Elmegreen \& Elmegreen (1987) to remove AC 10 and 11, namely barred galaxies and galaxies with companions, respectively. Elmegreen \& Elmegreen (1987) 
showed that grand design galaxies are larger than flocculent galaxies from an analysis of 762 spiral galaxies (selected from RC2) that are larger than $1^{\prime}$ with inclination less than $60^{\circ}$. They confirmed the preference of grand design galaxies to reside in dense groups found by Elmegreen \& Elmegreen (1982). Elmegreen \& Elmegreen (1989) applied a statistical correction to the Elmegreen \& Elmegreen (1987) sample. They showed that the grand design fraction is affected by the presence of the bar for early-type spirals, while for intermediate and late-type galaxies the grand design fraction is marginally affected by the presence of a bar, namely, nearly constant grand design fractions $\sim 0.7$ and $\sim 0.05$ for intermediate and late type galaxies, respectively.

There is only a small difference in neutral hydrogen content (Romanishin 1985), star formation rate (Elmegreen \& Elmegreen 1986), supernova rate (McCall \& Schmist 1986), CO surface brightness (Stark, Elmegreen, \& Chance 1987), and radio and X-ray emissions (Giuricin, Mardirossian, \& Mezzetti 1989) between grand design galaxies and flocculent galaxies; instead, there is a significant difference in the rotation curves of those two types of galaxies: grand design galaxies tend to have flat rotation curves while flocculent galaxies are likely to have steep rotation curves (Elmegreen 1990; Biviano et al. 1991). The dependence of arm classes on the shape of the rotation curve is due to the dependence of the arm multiplicity on the disk-to-halo ratio (Athanassoula, Bosma, \& Papaionnou 1987). Hence, it seems likely that arm structures do not depend significantly on internal properties, which are closely related to star formation rates, but instead on the disk-to-halo mass ratio.

The purpose of the present study is to understand the correlations between spiral arm classes and physical properties of galaxies, as well as global morphologies represented by Hubble types and bar types: this is achieved using a homogeneous flux limited sample of nearby galaxies provided by the SDSS DR7. Since the number of galaxies in the present analysis is more than twice larger than previous studies, we expect a better statistics for the frequency distribution and for correlation studies. We briefly discuss the environment dependence of the spiral arm morphology using the local background density derived from the nearest neighbor method. This issue will be addressed in detail in a forthcoming paper (Ann 2013). In Section 2, we describe the observational data and the method for the arm classification. The internal and external properties of spiral galaxies in relation with the spiral arm morphology is examined in Section 3. Summary and conclusions are provided in the last section.

\section{DATA AND MORPHOLOGY}

\subsection{Observational Sample}

We use a sample of nearby galaxies $(z<0.02)$ extracted from the Korea Institute for Advanced Study
Value Added Galaxy Catalog (KIAS VAGC) which provides some physical parameters such as the morphological types determined by an automated classifier (Park \& Choi 2005) and the concentration index along with coordinates, redshift, magnitudes and colors, etc. (Choi, Han, \& Kim 2010). Since the KIAS VAGC is based on the SDSS DR7, the sky coverage is $2.584 \mathrm{sr}$.

We expect the catalog to be almost complete up to $M_{r} \approx-16.1$ for galaxies with redshift less than $\mathrm{z}=0.02$, because it includes most of the bright galaxies excluded in the spectroscopic target galaxies. The number of galaxies extracted from the KIAS VAGC is 13762. From those, we selected a flux-limited sample of 1912 spiral galaxies with a Petrosian radius in $r$ band greater than $10^{\prime \prime}$ and inclination less than $65^{\circ}$. Since the flux-limited sample is biased (i.e., Malmquist bias), we made a volume-limited sample of 1725 spiral galaxies brighter than $M_{r}=-16.1$ by considering the observation limit of the SDSS, $r=17.7$. Because most of the spiral galaxies are known to be brighter than $M_{r} \approx=-16$, the present sample of galaxies includes most of the spiral galaxies within $z=0.02$. We used the previous volume-limited sample in the following analyses, except for the color-magnitude diagram (Fig. 3) in order to show which arm class is most affected by the Malmquist bias.

\subsection{Spiral Arm Classification}

We determined the morphology of the galaxies by visual inspection, using color images provided by the SDSS. We divided the spiral arms into three types: flocculent (F), multiple-arm (M), and grand design (G). We used the definition of arm classes of Elmegreen \& Elmegreen $(1982,1987)$ to determine the arm types. We assigned AC 1-4 to flocculent galaxies, AC 5-9 to multiple-arm galaxies, and AC 12 to grand design galaxies.

Flocculent galaxies (AC 1-4) are characterized by fragmented arms with no symmetry. A typical example is NGC 2841, classified as AC 3, which shows a fleece-like appearance of spiral patches. Grand design galaxies are characterized by two symmetric arms like those in M81. Multiple-arm galaxies are distinguished from grand design galaxies by the additional multiple arms (up to $\sim 10$ ) in the outer parts with two short symmetric arms in the inner parts. A good example of multiple-arm galaxies is M101. The Milky Way is also thought to be a multiple-arm galaxy since it has at least four long arms, with two arms connected to the end of the bar.

We distinguished barred galaxies (SAB and $\mathrm{SB}$ ) from non-barred galaxies (SA). We used bright galaxies from NED as a training sample for visual inspection. We also determined the early, intermediate, and late stage of Hubble type by visual inspection. We presented the catalog of arm classes for 1912 spiral galax- 


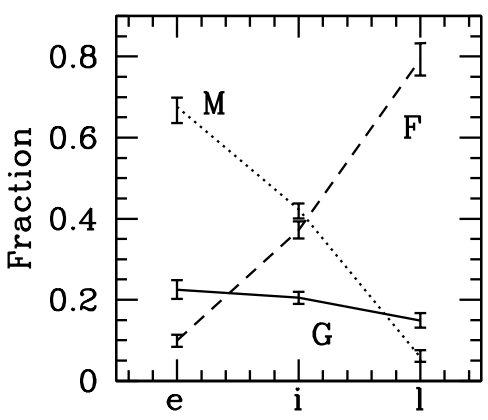

Fig. 1. - Fractional frequency distribution of arm classes as a function of the Hubble type. Solid, dotted, and shortdashed lines represent the grand design, multiple-arm, and flocculent arm, respectively.

ies on the web*. In Table 1, we present the number of galaxies and the relative frequency of arm types as a function of the Hubble type. We designated the early types of $0 / a$ to ab as 'e', b to cd as 'i', and d to $\mathrm{m}$ as 'l', respectively. Since galaxies in the present paper constitute a volume-limited sample $\left(M_{r}<-16.1\right)$, we did not apply the distance correction for the relative frequencies as done in Elmegreen \& Elmegreen (1989).

\section{RESULTS}

\subsection{Frequency Distribution of the Arm Classes}

Fig. 1 shows the relative frequency of spiral arm classes as a function of the Hubble type. Grand design spirals show nearly constant fractions along the Hubble type with a slight decrease in late-type spirals, whereas the fractions of multiple-arm and flocculent galaxies vary greatly. About $70 \%$ of early-type spirals have multiple arms and this fraction becomes $\sim 5 \%$ for late-type galaxies, whereas the fraction of flocculent arms is $\sim 10 \%$ in early-type galaxies and increases monotonically along the Hubble type. It becomes $\sim 80 \%$ for late type galaxies. Hence, late-type spirals are dominated by flocculent spirals.

As shown in Fig. 1, the relative frequency of multiple-arm and flocculent galaxies is very similar for intermediate-type galaxies. The dependence of the arm classes on Hubble type is in agreement with the results of Elmegreen \& Elmegreen (1989) but there are some differences in the distributions.

On the opposite, the dependence of arm classes on bar type, as shown in Fig. 2, is considerably different from the one shown in Fig. 1. Here, it is clear that flocculent arms are the most preponderant types of spiral structures, and that grand design arms are the rarest ones, especially in late type spiral galaxies. The fraction of flocculent galaxies decreases from SA to SAB to SB while that of grand design galaxies increases from

*http://earth.es.pusan.ac.kr/hbann/jkas/v46/AC

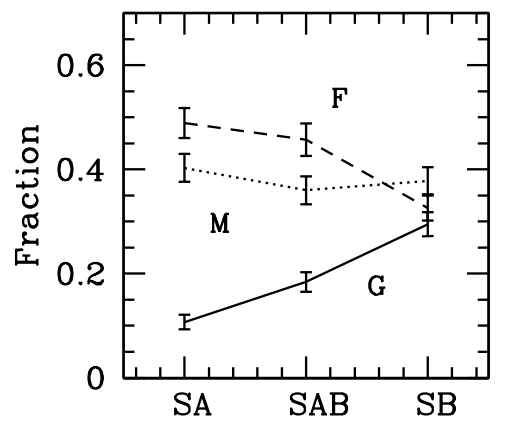

Fig. 2.- Fractional frequency distribution of arm classes as a function of bar type. Solid, dotted, and short dashed lines represent grand design, multiple-arm, and flocculent arm, respectively.

SA to SAB to SB. Multiple-arm galaxies show nearly constant fractions, regardless of the bar type.

To see the detailed dependence of the frequency of arm classes on Hubble and bar type in Table 1, we present the number and fraction of arm classes as a function of Hubble and bar types. The highest fraction of grand design arms is observed in SB galaxies with intermediate Hubble type, while the lowest grand design fraction is observed in late-type SA galaxies. On the other hand, flocculent arms are most preponderant in late-type SA galaxies, while they are least observed in early-type SB galaxies. In case of multiple arms, regardless of the bar type, they are predominant in early-type spirals, while their fraction is lowest $(\sim 5 \%)$ in late type spirals.

The fractions of bar types in the present sample are $0.35,0.30$ and 0.35 for $\mathrm{SA}, \mathrm{SAB}$, and $\mathrm{SB}$, respectively. They are similar to values in previous studies based on visual classification of galaxies using photographic plates (Elmegreen \& Elmegreen 1989; de Vaucouleurs, de Vaucouleurs, \& Corwin 1991). The fraction of strongly barred galaxies (SB) is also similar to that in the Revised Shapley-Ames Catalog of Bright Galaxies (Sandage \& Tammann 1981). However, as

Table 1.

Relative frequency of arm classes for SA, SAB and SB galaxies

\begin{tabular}{cccccccc}
\hline Hubble type & \multicolumn{3}{c}{ number } & \multicolumn{3}{c}{ fraction } \\
\hline & & $\mathrm{F}$ & $\mathrm{M}$ & $\mathrm{G}$ & $\mathrm{F}$ & $\mathrm{M}$ & $\mathrm{G}$ \\
\hline SA & $\mathrm{e}$ & 23 & 77 & 10 & 0.21 & 0.70 & 0.09 \\
& $\mathrm{i}$ & 148 & 144 & 48 & 0.44 & 0.42 & 0.14 \\
& $\mathrm{I}$ & 109 & 8 & 3 & 0.91 & 0.07 & 0.03 \\
$\mathrm{SAB}$ & $\mathrm{e}$ & 7 & 47 & 18 & 0.10 & 0.65 & 0.25 \\
& $\mathrm{i}$ & 91 & 118 & 52 & 0.35 & 0.45 & 0.20 \\
& $\mathrm{I}$ & 123 & 9 & 19 & 0.81 & 0.06 & 0.13 \\
$\mathrm{SB}$ & $\mathrm{e}$ & 8 & 123 & 51 & 0.04 & 0.68 & 0.28 \\
& $\mathrm{i}$ & 51 & 86 & 70 & 0.25 & 0.42 & 0.34 \\
& $\mathrm{I}$ & 132 & 10 & 50 & 0.69 & 0.05 & 0.26 \\
\hline
\end{tabular}




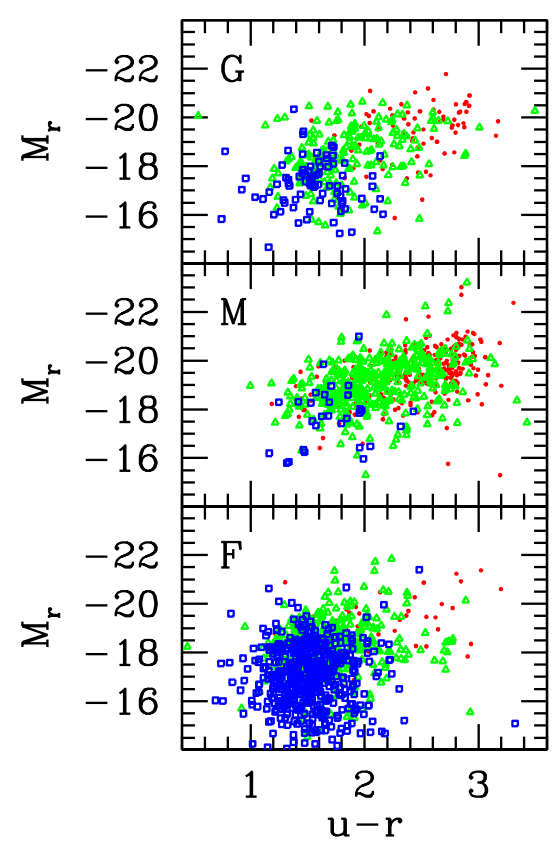

Fig. 3. - $M_{r}$ versus u-r diagram of 1912 spiral galaxies. Early type galaxies are represented by solid circles, while intermediate and late type galaxies are plotted as triangles and rectangles, respectively. The top panel shows grand design galaxies, middle and bottom panels display multiplearm and flocculent galaxies, respectively.

discussed by Lee et al. (2012), the fractions of barred galaxies in the literature are quite different if one considers studies of nearby galaxies in optical wave-bands. The reason for the discrepancy is partly due to the different methods of bar identification (visual or automated), and to different criteria for bar types. For example, the definition of strong bars in the Third Reference Catalogue of Bright Galaxies (de Vaucouleurs, de Vaucouleurs, \& Corwin 1991, hereafter RC3) is slightly different from those of Nair \& Abraham (2010) and Lee et al. (2012). Our definition of strong bar is close to that of RC3, because we used bright galaxies in the NED as a training sample with morphological types mostly adopted from the RC3. This is the reason for the slight difference in the bar fractions between our catalog and those which include the bar fractions derived from the SDSS galaxies by visual inspection (Nair \& Abraham 2010; Lee et al. 2012).

\subsection{Luminosity and Color}

Fig. 3 shows the $u-r$ versus $M_{r}$ diagram for a fluxlimited sample of spiral galaxies brighter than $r=17.7$, with redshift less than $z=0.02 . \quad M_{r}$ is the $r$-band absolute Petrosian magnitude normalized to $\mathrm{z}=0.1$ using co-moving distances with $\Omega_{m}=0.26, \Omega_{\Lambda}=0.74$, and $\mathrm{H}=100 \mathrm{~km} / \mathrm{s} / \mathrm{Mpc}$ (see Choi, Han, \& Kim 2010, for a detailed description). Grand galaxies $(\mathrm{G})$, multiplearm galaxies (M), and flocculent galaxies (F) are plot-
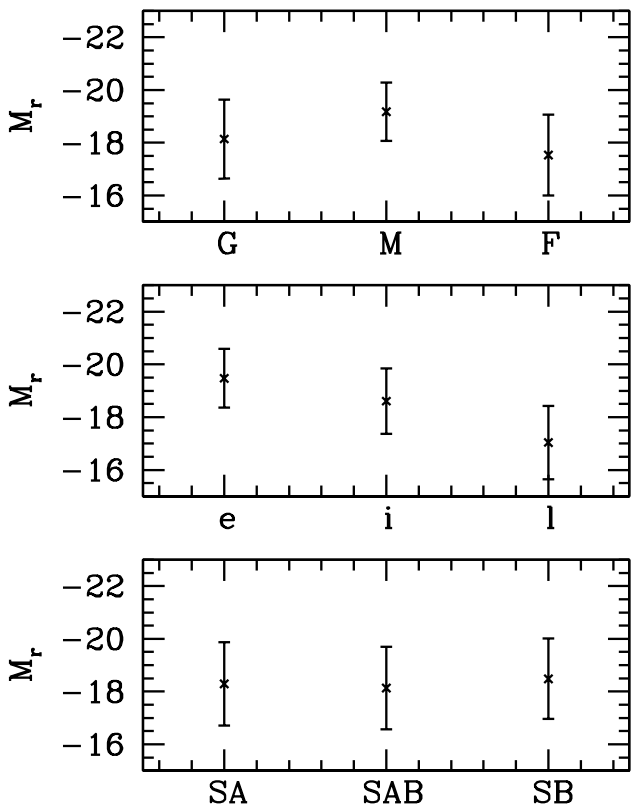

Fig. 4.- Luminosity of spiral galaxies. The top panel shows the luminosity distribution as a function of spiral arm types, the middle panel shows the luminosity distribution as a function of the openness of the spiral arm (Hubble type), and the bottom panel shows the luminosity distribution as a function of bar type.

ted in the top panel, middle panel and bottom panel, respectively. We distinguish the Hubble types by three colors, red (early-type), green (intermediate type) and blue (late-type). There seems to be a correlation between $M_{r}$ and $u-r$; the brighter the luminosity of a galaxy, the redder the color. However, the correlation between luminosity and color of a galaxy breaks down for flocculent arms if we only consider galaxies brighter than $M_{r} \approx-18$. The faint spiral galaxies with flocculent arms have a narrow range of $u-r$ colors, i.e., $1<u-r<2$ and show a large spread in luminosity for a given color. Most of the galaxies fainter than the limiting magnitude of the volumelimited sample $\left(M_{r}=-16.1\right)$ are flocculent. Hence, if we use flux-limited sample, the relative frequencies of arm classes are affected by the Malmquist bias although the mean luminosity and colors of both grand design and multiple-arm galaxies are almost unaffected.

The correlation between luminosity and color of spiral galaxies shown in Fig. 3 is a general property of galaxies, related to their formation history. As shown in Fig. 3, early-type spirals are likely to have redder colors while late-type spirals tend to have blue colors, regardless of their arm classes. This correlation between morphology and color can be extended to lenticular and elliptical galaxies, and makes the color of a galaxy a good proxy of morphology. The previous correlation seems to be closely related to the morphologydensity relation of galaxies (Dressler 1980) because the 


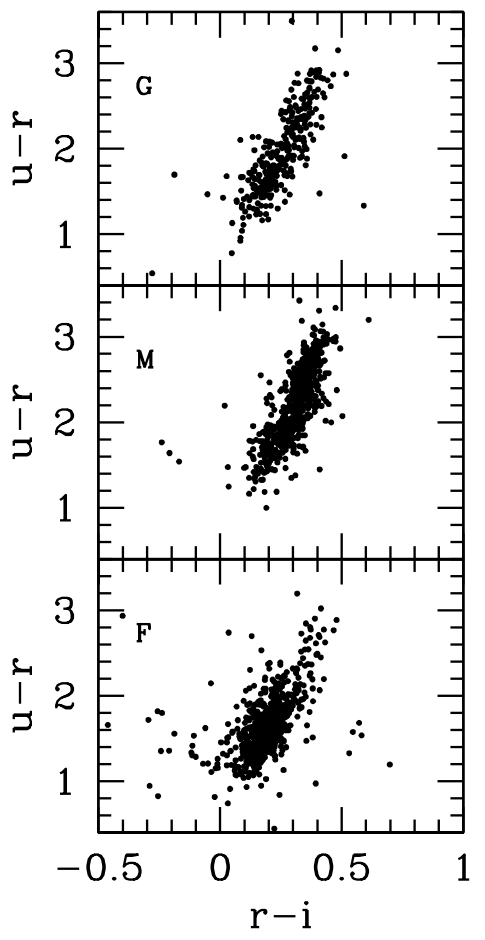

Fig. 5. $-u-r$ versus $r-i$ diagram of spiral galaxies.

star formation rate depends on the collapsing time scale $\tau \sim(G \rho)^{-1 / 2}$, which is inversely proportional to the local background density. Galaxies formed in dense regions are likely to have high star formation rate in the early stage of evolution due to short collapsing time. This leads to the formation of bulge-dominated spiral or elliptical galaxies in dense regions, and of bulgeless spirals or irregular galaxies in less dense regions.

Fig. 4 shows the mean luminosity $\left(M_{r}\right)$ of spiral galaxies sorted by arm class (top panel), Hubble type (middle panel), and bar type (bottom panel), respectively. The error bars represent the standard deviations of the mean absolute magnitudes. It is clear that the luminosity of spiral galaxies does not depend much on the bar type, but there is a strong correlation between the luminosity and Hubble type. The luminosity dependence of arm classes is not as strong as that of the Hubble type. However, flocculent spirals are fainter than grand design and multiple-arm spirals and, on average, multiple-arm spirals are more luminous. The origin of the luminosity dependence of arm classes, i.e., higher luminosities of multiple-arm galaxies and lower luminosity of flocculent galaxies, is not well-understood.

If we consider the arm class, Hubble type, and bar type together, the most luminous galaxies are those classified as early, multiple-arm, and barred, while the faintest galaxies are those classified as late, flocculent, non-barred. The presence of bars does not affect the luminosity of a galaxy significantly except for late type flocculent arms; for this case SB galaxies are $\sim 1 \mathrm{mag}$

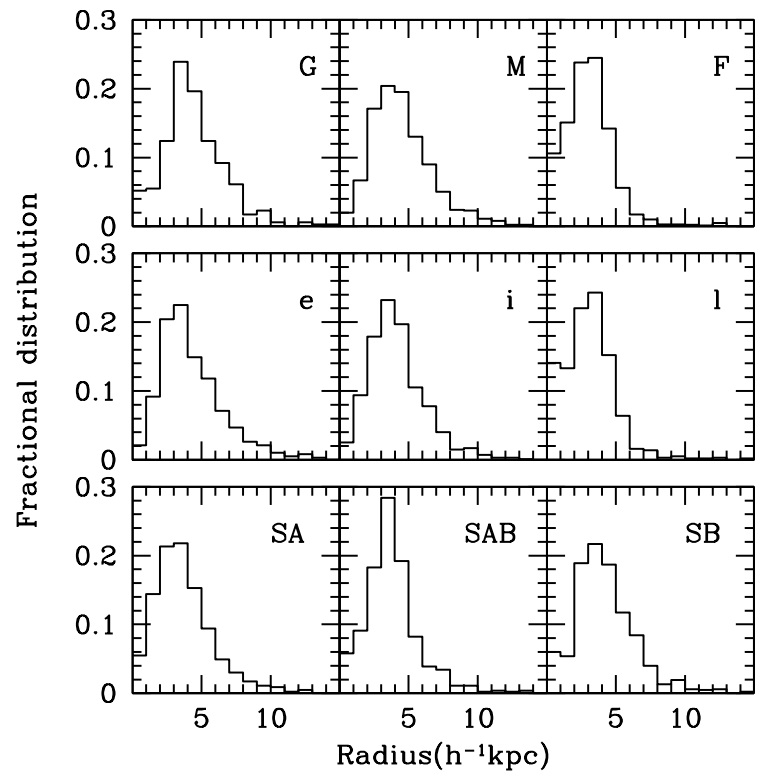

Fig. 6. - Petrosian radius of spiral galaxies. Top, middle, and bottom panels show the fractional distribution of the Petrosian radius for arm classes, Hubble types, and bar types, respectively.

brighter than SA galaxies. Multiple-arm galaxies are always brighter than the other types, except for late type SAB galaxies; grand design are $\sim 0.1$ mag brighter than multiple-arm galaxies.

Fig. 5 shows the $u-r$ versus $r-i$ diagram of spiral galaxies sorted by arm class, grand design spirals are in the top panel, multiple-arm spirals in the middle panel, and flocculent spirals in the bottom one. There is not much difference in the color range between the grand design spirals and multiple-arm spirals. However, for flocculent spirals, the majority of galaxies has colors $u-r<2.2$ and $r-i<0.3$. The preponderance of flocculent spirals with bluer colors seems to be related to the fact that the majority of flocculent spirals are late-type spirals which are thought to have more gas content than early-type spirals. Since galaxy colors reflect the star formation history, as well as the current star formation rate, flocculent spirals are supposed to have a formation history different from that of grand design and multiple-arm galaxies.

The similarity of colors between grand design and multiple-arm galaxies, and the dissimilarity between flocculent galaxies and other types, are related to different driving mechanisms of the spiral structures. The spiral structures of flocculent galaxies are caused by the local gravitational instability propagated by stochastic processes (Gerola \& Seiden 1978; Seiden \& Gerola 1982), whereas those of grand design and multiple-arm galaxies by the global gravitational instability driven by density waves (Lin \& Shu 1964). Since grand design and multiple-arm spirals are brighter than floccu- 
Table 2.

Mean radii of spiral galaxies

\begin{tabular}{cccccccccc}
\hline & \multicolumn{3}{c}{ arm class } & \multicolumn{3}{c}{ Hubble stage } & \multicolumn{3}{c}{ bar type } \\
\hline & $\mathrm{G}$ & $\mathrm{M}$ & $\mathrm{F}$ & $\mathrm{e}$ & $\mathrm{i}$ & $\mathrm{l}$ & $\mathrm{SA}$ & $\mathrm{SAB}$ & $\mathrm{SB}$ \\
\hline $\mathrm{R}\left(h^{-1} \mathrm{kpc}\right)$ & 4.1 & 4.4 & 3.2 & 4.2 & 4.1 & 3.3 & 3.8 & 3.8 & 4.2 \\
$\sigma_{R}\left(h^{-1} \mathrm{kpc}\right)$ & \pm 2.2 & \pm 2.2 & \pm 1.7 & \pm 2.2 & \pm 2.0 & \pm 1.9 & \pm 2.1 & \pm 1.9 & \pm 2.1 \\
$\mathrm{~N}$ & 327 & 648 & 750 & 377 & 860 & 488 & 570 & 484 & 581 \\
\hline
\end{tabular}

Table 3.

Concentration index of spiral galaxies

\begin{tabular}{cccccccccc}
\hline & \multicolumn{3}{c}{ arm class } & \multicolumn{3}{c}{ Hubble stage } & \multicolumn{3}{c}{ bar strength } \\
\hline & $\mathrm{G}$ & $\mathrm{M}$ & $\mathrm{F}$ & $\mathrm{e}$ & $\mathrm{i}$ & $\mathrm{l}$ & $\mathrm{SA}$ & $\mathrm{SAB}$ & $\mathrm{SB}$ \\
\hline $\mathbf{C}$ & 2.32 & 2.41 & 2.21 & 2.61 & 2.26 & 2.15 & 2.30 & 2.25 & 2.33 \\
$\sigma_{\mathbf{C}}$ & \pm 0.36 & \pm 0.40 & \pm 0.30 & \pm 0.41 & \pm 0.31 & \pm 0.27 & \pm 0.36 & \pm 0.36 & \pm 0.38 \\
\hline
\end{tabular}

lent spirals, global density waves are driven in massive disks. This is why dwarf spirals with grand design and multiple arms are so rare in the local Universe. On the other hand, a non-negligible fraction of flocculent spirals, in particular those bluer than $u-r \approx 2$ are likely to be dwarf spirals.

\subsection{Size}

In Fig. 6, we plot the fractional distribution of radii of spiral galaxies sorted by arm class (top panels), Hubble type (middle panels), and bar type (bottom panels). We used the Petrosian radius $\left(R_{\text {pet }}\right)$ provided by the SDSS for the representative size of a galaxy. The general shape of the frequency distributions is characterized by an asymmetric distribution with a well-defined peak, i.e., steep declines to a smaller size and shallow declines to a larger size. However, there are some differences in the distributions. For galaxies grouped by arm class, the frequency distribution of flocculent spirals shows a narrow peak with a marginal extension to larger galaxies. The fraction of galaxies larger than $R_{\text {pet }}=7 h^{-1} \mathrm{kpc}$ is $\sim 2 \%$ only. It is quite different from the frequency distribution of grand design and multiple-arm spirals, which shows considerable extensions to a larger radius. Grand design and multiple-arm spirals have $\sim 10 \%$ of galaxies with $R_{\text {pet }}$ larger than 7 $h^{-1} \mathrm{kpc}$, The mean radii of grand design, multiple-arm, and flocculent spirals are $(3.6 \pm 2.3) h^{-1} \mathrm{kpc},(4.2 \pm 2.3)$ $h^{-1} \mathrm{kpc}$, and $(2.7 \pm 1.8) h^{-1} \mathrm{kpc}$, respectively. Similar distributions are found for galaxies divided by Hubble type. Here, late-type galaxies show the narrowest distribution, with a mean radius of $2.9 \pm 1.9 h^{-1} \mathrm{kpc}$. The frequency distributions of early and intermediate type spirals show extensions to larger radii. In the case of bar type, however, there is not much difference between the frequency distributions of non-barred and barred galaxies.

We summarize in Table 2 the values of radii and standard deviations of spiral galaxies sorted by arm class, Hubble type and bar type. It is clear that early-

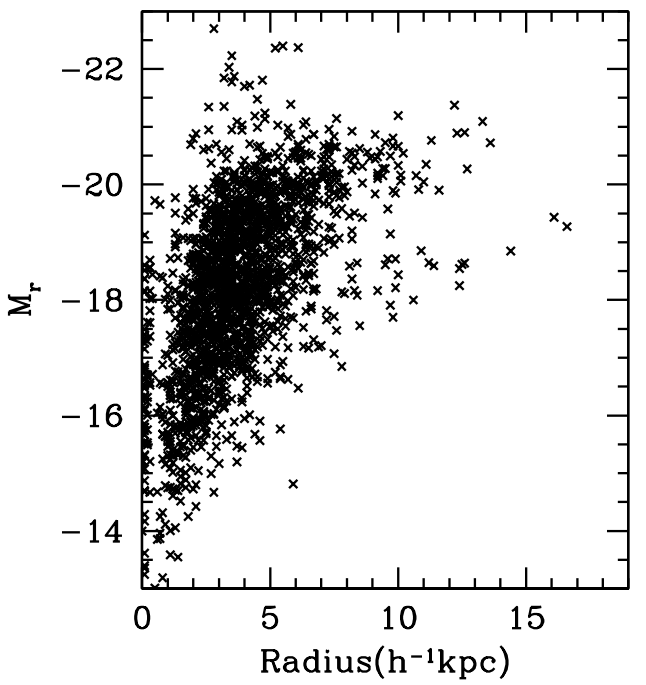

Fig. 7.- Correlation between $M_{r}$ and Petrosian radius $\left(R_{p e t}\right)$.

type grand design galaxies seems to have the largest radii, while late-type flocculent galaxies have the smallest ones.

There seem to be some restrictions on the size of a galaxy that drives the global density waves. A large fraction of grand design galaxies is larger than $R_{\text {pet }}=5 h^{-1} \mathrm{kpc}$ while most of the flocculent galaxies are smaller than $R_{\text {pet }}=5 h^{-1} \mathrm{kpc}$.

Since it is well known that luminous galaxies are larger than the fainter ones, the dependence of spiral arm class on galaxy size seems to originate from the correlation of spiral arm morphology with the luminosity. Fig. 7 shows the distribution of $M_{r}$ as a function of the Petrosian radius. As expected, there is a tight correlation between luminosity and radius, but some galaxies $(\sim 10 \%)$ show larger sizes for their luminosity. There seems to be an upper limit for the galaxy size at 

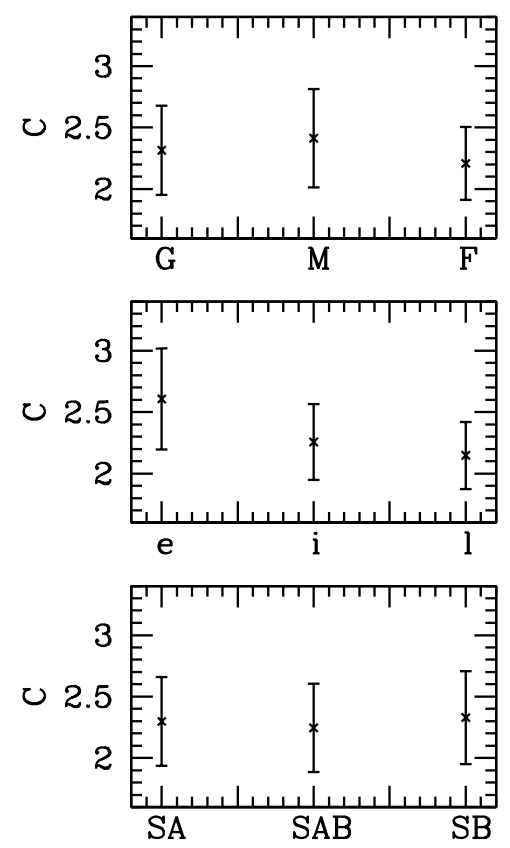

Fig. 8. - Concentration index as a function of arm classes, Hubble types and bar types.

a given luminosity.

\subsection{Concentration Index}

The concentration index $\mathbf{C}$ is a parameter which quantifies the galaxy luminosity. We calculated the value of $\mathbf{C}$ using two Petrosian radii, $R_{\text {pet,90 }}$ and $R_{\text {pet }, 50, \text { as }}$

$$
\mathbf{C}=R_{\text {pet }, 90} / R_{\text {pet }, 50}
$$

where $R_{p e t, 90}$ and $R_{p e t, 50}$ are the radii within which $90 \%$ and $50 \%$ of the galaxy flux is emitted. We list the mean concentration index of galaxies divided by arm class, Hubble type and bar type in Table 3. The mean values are obtained with a 3 -sigma rejection method to suppress the effect of highly deviant values. The concentration index decreases from early-type galaxies to late types, but there is no distinct trend for the bar type. The large $\mathbf{C}$ in early-type galaxies is due to their large bulge. There is no strong correlation between concentration index and spiral arm type. However, on average, galaxies with grand design arms have a large concentration index than those with flocculent arms. The reason is due to the preponderance of flocculent galaxies in the late-type sample.

\subsection{Local Background Density}

There are several ways to define a parameter which measures the local background density which affects the structure of a galaxy. For example, the local background density based on the distance to the $n$th nearest

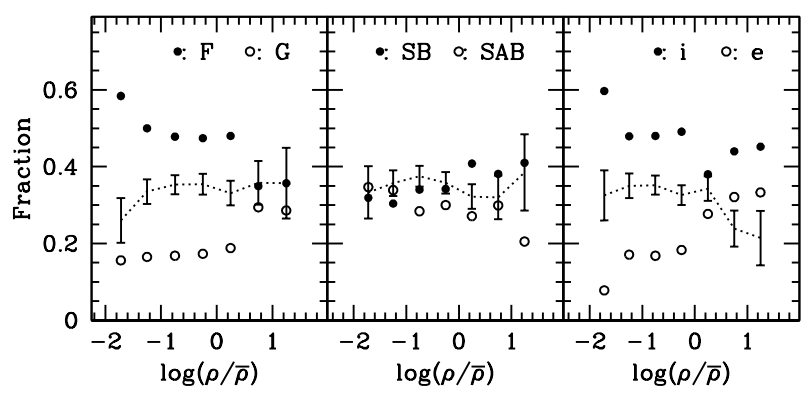

Fig. 9. - ractional distributions of arm class, Hubble type and bar type as a function of the local background density. The left panel shows the distributions of arm classes $(\mathrm{G}$, $\mathrm{M}, \mathrm{F}$ ), the middle panel displays those of bar types (SA, $\mathrm{SAB}, \mathrm{SB}$ ), and the right panel plots the Hubble types (e, i, l). The dotted lines indicate $\mathrm{M}$ in the left panel, SA in the middle panel and $l$ in the right panel, respectively.

neighbor has been widely used (see Park et al. 2007). Following a recent analysis of the measures of galaxy environment by Muldrew et al. (2012) who showed that the local background density using the $n$th nearest neighbor with small $n$ is one of the best measures of the local environment, we calculated the local background density $\rho$ using the projected distance to the 5 th nearest neighbor.

The local background density using the $n$th nearest neighbor can be defined as

$$
\rho=\frac{5}{4 \pi r_{p}^{2}},
$$

where $r_{p}$ is the projected distance to the 5 th nearest galaxy brighter than $M_{r}=M^{*}$ with velocity difference between the target galaxy and neighbor $(|\Delta V|)$ less than $\Delta V^{*}=1000 \mathrm{~km} / \mathrm{s}$. The natural choice for the luminosity constraint $M^{*}$ is the limiting magnitude that defines the volume limited sample of the present analysis. Since our sample is drawn from the SDSS, we used $M_{r}=-16.12$ as $M^{*}$ which corresponds to the $M_{r}$ of a galaxy with $r=17.7$ at $z=0.02$. As the surface number density depends on the number of stars to be searched $(n)$ along with $M^{*}$ and $\Delta V^{*}$, we used a normalized quantity $\rho / \bar{\rho}$ for the local background density of a target galaxy where $\bar{\rho}$ is the mean value of $\rho$.

Fig. 9 shows the fractional distributions of spiral galaxies sorted by their spiral arm class (G, M, F), bar type (SA, SAB, SB) and Hubble type (e, i, l) as a function of the local background density. There seems to be some dependence of the arm class, Hubble type and bar type on the local background density although the degrees of dependency are different. Among the three morphological properties, the Hubble type shows the strongest correlation with the local background density and the bar type the weakest. The fraction of early type spiral increases steeply with increasing local background density while that of intermediate and late type galaxies decreases with density. This means that 
the morphology-density relation (Dressler 1980), well established for the broad morphological types (ellipticals, lenticulars, spirals), seems to hold within spiral galaxies. It is worth mentioning that the morphology of intermediate Hubble types (b, bc, c, cd) is closer to late Hubble types than early Hubble types with respect to the morphology-density relation.

The density dependence of bar type, as shown in the middle panel of Fig. 9, is interesting. The fraction of SA galaxies is nearly constant regardless of the local background density, whereas SB and SAB galaxies show opposite trend in the sense that the fraction of SB galaxies increases with density. However, if we consider the large errors of the fractions in the extreme densities where the numbers of galaxies are too small for good statistics, there is no significant correlation between the bar strength and the local background density.

In case of arm classes, as shown in the left panel of Fig. 9, there is a clear trend in the density dependence of arm classes. The fraction of grand design galaxies increase with the local background density while that of flocculent galaxies decreases, with nearly constant fractions for multiple-arm galaxies. Hence, our result of the density dependence of arm classes agrees with Elmegreen \& Elmegreen $(1982,1987)$ who found that grand design galaxies are more frequent in high density environment. The density dependence of spiral arm classes is also consistent with that observed in the cluster spirals (Choi \& Ann 2011) which showed that the fraction of flocculent spirals increases with increasing cluster-centric radius.

\section{SUMMARY AND CONCLUSIONS}

We classified the arm class, Hubble type, and bar type (SA, SAB, SB) of 1912 nearby galaxies $(z<0.02)$ using the color images of the SDSS DR7 by visual inspections. We constructed the present sample from the KIAS VAGC (Choi, Han, \& Kim 2010). The sample size of our analysis is more than twice larger than the earlier ones (Elmegreen \& Elmegreen 1982, 1987) and almost complete to $z=0.02$ for galaxies in the Northern hemisphere. We presented the basic statistics of the arm classes, Hubble types and bar types along with a complete catalog of arm classes, broad Hubble types, and bar types.

The majority of early-type spirals has multiple arms ( 70\%), while flocculent arms are most frequent in late-type spirals $(\sim 80 \%)$. Grand design fractions are nearly constant along the Hubble types. The dependence of arm classes on the bar type is much different from that on the Hubble types. The fraction of grand design galaxies increases from $\mathrm{SA}$ to $\mathrm{SAB}$ to $\mathrm{AB}$ while that of multiple-arm galaxies decreases from SA to SAB to SB. Multiple-arm galaxies show nearly constant fraction, regardless of bar type. If we consider the Hubble and bar types together, the late-type non-barred galaxies with flocculent arms are most preponderant. They constitute $\sim 30 \%$ of nearby spirals. This fraction is about three times larger than the mean fractions.

There are small differences in the mean absolute magnitudes of the three arm classes: $M_{r}=-18.4 \pm 1.2$, $M_{r}=-19.2 \pm 1.0$, and $M_{r}=-18.0 \pm 1.1$ for the grand design, multiple-arm, and flocculent galaxies, respectively. The fainter luminosity of flocculent galaxies is related to the preponderance of late-type spirals in the flocculent galaxies since late-type spirals are fainter and smaller than early types. If we consider the arm class and Hubble type together, early-type multiple-arm galaxies have the largest mean radius of $4.3 \pm 2.1) h^{-1} \mathrm{kpc}$ and late-type flocculent spirals have the smallest mean radius of $3.2 \pm 1.8) h^{-1} \mathrm{kpc}$. The fraction of flocculent spirals larger than $7 h^{-1} \mathrm{kpc}$ is $\sim 0.03$, while that of grand design spirals is $\sim 0.12$. The fraction of multiple-arm spirals is similar to that of grand design spirals.

The color distribution of the flocculent galaxies is much different from that of the grand design and multiple-arm galaxies. On average, it is $\sim 0.3 \mathrm{mag}$ and $\sim 0.6 \mathrm{mag}$ bluer than grand design galaxies and multiple-arm galaxies, respectively, in $u-r$ colors.

There is a clear difference in the environment of galaxies that have different spiral structures, such as arm class and Hubble type. Grand design galaxies and early type galaxies are likely to be found in high density environment while flocculent galaxies and late type galaxies are likely to be observed in the low-density environment. Hence, the density dependence of arm class and Hubble type is in agreement with earlier findings (Elmegreen \& Elmegreen 1982, 1987). The density dependence of bar type is weaker than that of arm class and Hubble type with a tendency of strong bars in high density regions.

\section{ACKNOWLEDGMENTS}

This work is supported in part by the the National Research Foundation of Korea (NRF) research grant 2010-0023319.

\section{REFERENCES}

Ann, H. B. 2013, in preparation

Athanassoula, E., Bosma, A., \& Papaioannou, S. 1987, Halo Parameters of Spiral Galaxies, A\&A, 179, 23

Bertin, G., Lin, C. C., Lowe, S. A., \& Thurstans, R. P. 1989, Modal Approach to the Morphology of Spiral Galaxies. I - Basic Structure and Astrophysical Viability, ApJ, 338, 738

Bertin, G., Lin, C. C., Lowe, S. A., \& Thurstans, R. P. 1989, Modal Approach to the Morphology of Spiral Galaxies. I - Basic Structure and Astrophysical Viability, ApJ, 338, 1048

Biviano, A., Girardi, M., Giuricin, G., Mardirossian, F., \& Mezzetti, M. 1991, Arm Classification and Velocity Gradients in Spiral Galaxies, ApJ, 376, 458 
Choi, I. Y.-G., \& Ann, H. B. 2011, Spiral Arm Morphology in Cluster Environment, JKAS, 44, 161

Choi, Y.-Y., Han, D.-H., \& Kim, S. S. 2010, Korea Institute for Advanced Study Value-Added Galaxy Catalog, JKAS, 43, 191

de Vaucouleurs, G., de Vaucouleurs, A., \& Corwin, J. R. 1976, Second Reference Catalogue of Bright Galaxies (Austin: University of Texas Press)

de Vaucouleurs, G., de Vaucouleurs, A., Corwin, H. G. Jr., Buta, R. J., Paturel, G., \& Fouque, P. 1991, Third Reference Catalogue of Bright Galaxies (Austin: University of Texas Press)

Dressler, A. 1980, Galaxy Morphology in Rich Clusters - Implications for the Formation and Evolution of Galaxies, ApJ, 236, 351

Elmegreen, D. M., \& Elmegreen, B. G. 1982, Floccullent and Grand Design Spiral Structure in Field, Binary and Group Galaxies, MNRAS, 201, 1021

Elmegreen, D. M., Elmegreen, B. G., \& Dressler, A. 1982, Floccullent and Grand Design Spiral Arm Structure in Cluster Galaxies, MNRAS, 201, 1035

Elmegreen, B. G., \& Elmegreen, D. M. 1986, Do Density Waves Trigger Star Formation?, ApJ, 311, 554

Elmegreen, D. M., \& Elmegreen, B. G. 1987, Arm Classifications for Spiral Galaxies, ApJ, 314, 3

Elmegreen, B. G., \& Elmegreen, D. M. 1989, On the Relative Frequency of Flocculent and Grand Gesign Spiral Structures in Barred Galaxies, ApJ, 342, 677

Elmegreen, B. G. 1990, Grand Design, Multiple Arm, and Floccullent Spiral Galaxies, NYASA, 596, 40

Gerola, H., \& Seiden, P. E. 1978, Stochastic Star Formation and Spiral Structure of Galaxies, ApJ, 223, 129

Giuricin, G., Mardirossian, F., \& Mezzetti, M. 1989, Radio Continuum Emission and Arm Classification in Spiral Galaxies, A\&A, 208, 27

Giuricin, G., Monaco, P., Mardirossian, F., \& Mezzetti, M. 1994, The Local Galaxy Density and the Arm Class of Spiral Galaxies, ApJ, 425, 450

Goto, T., Yamauchi, C., Fujita, Y., Okamura, S., Sekiguchi, M., Smail, I., Bernardi, M., \& Gomez, P. L. 2003, The Morphology-Density Relation in the Sloan Digital Sky Survey, MNRAS, 346, 601

Lee, G.-H., Park, C., Lee, M. G., \& Choi, Y.-Y. 2012, Dependence of Barred Galaxy Fraction on Galaxy Properties and Environment, ApJ, 745, 125

Lin, C. C., \& Shu, F. H. 1964, On the Spiral Structure of Disk Galaxies, ApJ, 140, 646

McCall, M. L., \& Schmidt, F. H. 1986, Supernovae in Flocculent and Grand Design Spirals, ApJ, 311, 548

Nair, P. B.,\& Abraham, T. G. 2010, On the Fraction of Barred Spiral Galaxies, ApJ, 714, 260
Muldrew, S. I., et al. 2012, Measures of Galaxy Environment - I. What is 'Environment'?, MNRAS, 419, 2670

Park, C., \& Choi, Y.-Y. 2005, Morphology Segregation of Galaxies in Color-Color Gradient Space, ApJ, 635, L29

Park, C., Choi, Y.-Y., Vogeley, M. S., Gott, J. R. III., \& Blanton, M. R. 2007, Environmental Dependence of Properties of Galaxies in the Sloan Digital Sky Survey, ApJ, 658, 898

Romanishin, W. 1985, Star Formation in Grand Design and Flocculent Spiral Galaxies, ApJ, 289, 570

Sandage, A., \& Tammann, G. A. 1981, A Revised Shapley-Ames Catalog of Bright Galaxies (Washington: Carneigie Institution of Washington)

Seiden, P. E., \& Gerola, H. 1982, Propagating Star Formation and the Structure and Evolution of Galaxies, Fund. Cosmic Phys., 7, 241

Stark, A. A., Elmegreen, B. G., \& Chance, D. 1987, Molecules in Galaxies. V - CO Observations of Flocculent and Grand-Design Spirals, ApJ, 322, 64

Turner, E. L. 1976, Binary Galaxies. II - Dynamics and Mass-to-Light Ratios, ApJ, 209, 304

Turner, E. L., \& Gott, J. R. III., 1976, Groups of Galaxies. I. A Catalog, ApJS, 32, 409

van den Bergh, S. 1960, A Preliminary Luminosity Classification of Late-Type Galaxies, ApJ, 131, 215

van den Bergh, S. 1960, A Preliminary Luminosity Classification of Type Sb, ApJ, 131, 558 